\title{
GCU
}

Glasgow Caledonian

University

University for the Common Good

\section{Random neural network based cognitive-eNodeB deployment in LTE uplink}

Adeel, Ahsan; Larijani, Hadi; Ahmadinia, Ali

Published in:

2015 IEEE Global Communications Conference (GLOBECOM)

DOI:

10.1109/GLOCOM.2015.7417122

Publication date:

2016

Document Version

Author accepted manuscript

Link to publication in ResearchOnline

Citation for published version (Harvard):

Adeel, A, Larijani, H \& Ahmadinia, A 2016, Random neural network based cognitive-eNodeB deployment in LTE uplink. in 2015 IEEE Global Communications Conference (GLOBECOM). IEEE, San Diego, CA, pp. 1-7, IEEE Global Communications Conference (GLOBECOM), San Diego, United States, 6/12/15.

https://doi.org/10.1109/GLOCOM.2015.7417122

\section{General rights}

Copyright and moral rights for the publications made accessible in the public portal are retained by the authors and/or other copyright owners and it is a condition of accessing publications that users recognise and abide by the legal requirements associated with these rights.

Take down policy

If you believe that this document breaches copyright please view our takedown policy at https://edshare.gcu.ac.uk/id/eprint/5179 for details of how to contact us. 


\title{
Random Neural Network based Cognitive-eNodeB deployment in LTE uplink
}

\author{
Ahsan Adeel, Hadi Larijani, Ali Ahmadinia \\ School of Engineering \& Built Environment \\ Glasgow Caledonian University \\ Email: Ahsan.Adeel@gcu.ac.uk, H.Larijani@gcu.ac.uk, Ali.Ahmadinia@gcu.ac.uk
}

\begin{abstract}
Artificial intelligence (AI)/machine learning (ML) based cognitive solutions have widely been applied to deal with downlink inter-cell interference coordination (ICIC) in longterm evolution (LTE) systems. This paper presents a random neural network (RNN) based novel framework to improve ICIC and radio resource management (RRM) in LTE-Uplink system. The RNN based cognitive engine (CE) is embedded within the eNodeB which allocates optimal radio parameters to attached users and also suggests acceptable transmit power to users served by adjacent cells, in order to reduce inter-cell interference (ICI). The proposed CE concurrently achieves long-term learning, fast decision making, and less computational complexity. These three CE design features are essential for the development and practical deployment of any real-time cognitive communication system and most of the existing AI/ML based cognitive solutions in literature lack them. The performance of RNN based optimization framework is compared with artificial neural network (ANN) and stateof-the-art fractional power control (FPC) scheme. In six different test-cases, simulation results have shown an improvement of $\mathbf{5 3 . 8 8 \%} \mathbf{- 8 7 . 5 3 \%}$ in decision making accuracy and a decrease of $44.22 \%$ in scheduling delay as compared to ANN. In addition, throughput gain of $16.13 \%$ and $18.62 \%$ has been achieved as compared to ANN and FPC schemes respectively.
\end{abstract}

\section{INTRODUCTION}

In LTE uplink system, transmit power is one of the key and most influential parameters, which can address the challenges posed by channel fading, ICI, adjacent-channel interference (ACI), and user equipment (UE) excessive transmission power. 3rd Generation Partnership Project (3GPP) defined FPC for LTE uplink as an open loop power control (OLPC), which relies on the assumption that interference generated towards other cells is mostly because of cell-edge users. However, generated interference follows a different trend which shows that the assumption that users experiencing the lowest pathgain generates most of the interference is not always true [1]. This suggests to adjusting the power in order to compensate the generated interference rather than the path-gain.

In literature, ICIC and RRM schemes have been extensively studied and researchers have proposed solutions with variation in approaches from statistical, analytical, and classical network optimization schemes to self-organized approaches [2][3]. If the radio has complete or partial knowledge of operational electromagnetic environment, user requirements, and parameters effecting the reliability of communication, in advance, then providing services such as autonomous computing and optimization could be achievable. Therefore, the framework of cognitive or self-organizing network has been of great appeal for ICIC and RRM requiring minimum supervision. To make such design possible, researchers have borrowed ideas from $\mathrm{AI} / \mathrm{ML}$ techniques. In [4], the authors proposed an interference management scheme using cognitive base stations (CBSs) for LTE and discussed the insufficiency of traditional interference management schemes for isolated cell or a multi-cell LTE network. The authors argued that CBSs can exploit their knowledge of radio-scene to intelligently allocate resources and to mitigate prohibitive co-channel interference. The key required feature for such intelligent system is to have a good learning capability, i.e. the ability to learn well and behave intelligently, which is critical to the performance of autonomously deployed cognitive radios (CRs). Training or exploration is the task by which a CE gets through the process of learning a desired systems behaviour and capabilities. The training speed, accurate learning, available training samples, and computational complexity during this task are of paramount importance to the systems operational performance and also limiting factors for CR to achieve optimal configuration settings in real-time. Researchers have made great effort to solve the $\mathrm{CE}$ training problems and demonstrated that a $\mathrm{CE}$ can be trained in a reasonable amount of time and effort [5].

In the process of $\mathrm{CE}$ exploitation (testing), if the radio does not know any satisfactory solution to the current problem, then it will have no other option but to explore (train) again, which consumes time and energy. To avoid the process of retraining during radios operation, it is necessary to put the $\mathrm{CE}$ through all expected operating conditions during training phase. However, it is practically impossible to explore all possible conditions as a priori. Therefore, it is reasonable to assume that a $\mathrm{CE}$ is going to face an unknown condition sooner or later and may require retraining. What if in case, the radio is operating in a critical mission? then it may not have time to retrain again and again [5]. Consequently, the capability of long-term learning is of great importance which enable CEs to adapt themselves with respect to severe change in environment.

Insufficiency of long-term learning along with the concurrent achievement of fast decision making and less complexity are the major limiting factors of ANN and many other AI/ML approaches. Researchers have proposed both supervised and unsupervised learning algorithms for ICIC and RRM. The authors in [6] proposed a Q-learning based aggregate interference control scheme and presented a framework which 
combined reinforcement learning (RL) and ANN. However, ANN suffers from limited generalization, slow calculation rate at run-time, local minima, and over-fitting problems. Support vector regression for proactive resource allocation and stochastic-learning based gradient algorithm for adaptive power control is presented in [7][8]. However, no testing under CE design requirements is shown. Recently, the authors in [9] proposed a RL based decentralized solution for joint power control and cells association in heterogeneous networks. A game theory based combined power and interference control framework for LTE-downlink is presented in [10]. The approaches based on unsupervised learning schemes help CEs to work in alien environment because of their no a priori knowledge assumption. However, for realistic applications, the size of state-space could be so large that learning may take a long time and even become impossible in a reasonable amount of time frame. As a result, the generalization over the statespace is necessary, which is insufficient in RL. A limitation of the game theory approach is that it requires user-specific utility parameters, which can not always be acquired in many situations [2].

To the best of our knowledge, limited work has been conducted to concurrently achieve aforementioned CE design features. RNNs inherent properties such as: (a) easy and efficient computation (b) low complexity of standard learning algorithm (c) energy-efficient hardware implementation (d) less dependence on network structure (e) strong generalization capability even with small training dataset, makes RNN a better choice for $\mathrm{CE}$ design [11]. In our previous work [12][13], we addressed some of the CE exploration and exploitation challenges and presented the out-performance of RNN over ANN and hierarchical RNN over traditional RNN in terms of generalization, learning efficiency, and computational complexity. The work in [14][15] further addressed the convergence speed and local minima problems of gradient descent (GD) based RNN by implementing adaptive inertia weight particle swarm optimization (AIW-PSO), differential evolution (DE), and genetic algorithm (GA) training algorithms.

The main contributions of this paper are:

1) Extended our previous research by presenting RNNs first application to the problem of LTE uplink RRM and ICIC. When studying the interaction of ICIC with RRM mechanisms, very often the novelty is the modification of one RRM functionality in terms of channel allocation/frequency reuse pattern. Moreover, most of the practical implemented methods fail to comply with LTEs full frequency spectrum usage requirement. This paper presents a novel and simple framework which reduces ICI with zero bandwidth loss and jointly tackles both power and MCS selection. Whereas, most of the existing literature addressed the issues of power allocation, modulation and coding scheme (MCS) selection, and ICIC separately. The traditional FPC method has been replaced with a closed loop power control (CLPC) by embedding a $\mathrm{CE}$ in eNodeB. This replacement aims to schedule optimal transmit power and MCS to the attached user equipments' (UEs) as well as suggest acceptable transmit power to the UEs served by neighbouring cells. The user specific power adjustment has a centralized control at the base station (BS). The mobile station (MS) feeds back the channel quality information (CQI) to the BS, which calculates optimal uplink transmit power level and instructs the MS to transmit at that level. The basic idea is to control the power to compensate for the generated interference to the system rather than the path-gain.

2) Evaluated the proposed $\mathrm{CE}$ with respect to $\mathrm{CE}$ design requirements. Our proposed CR checks the feasibility and reliability of proposed system in real-time cases, where there can be training time constraints or where the retraining is generally not appropriate. The CE was trained with ANN and RNN using the dataset obtained from the system model. The training with ANN is to demonstrate the coped challenges taking its performance as a reference for RNN.

3) To minimize the cost function we used GD and levenberg marquardt (LM) for training. In literature, researchers have mostly considered only GD as a training algorithm for RNN and ANN comparisons.

4) The performance improvement of proposed optimization framework with ANN and RNN has been compared with state-of-the-art FPC scheme in terms of throughput and ICI.

\section{System Architecture}

\section{A. Model}

In Orthogonal Frequency-Division Multiple Access (OFDMA) systems, ICI can be considered as a collision between resource blocks (RBs) [16]. Fig.1 illustrates such collision model and the corresponding ICIC mechanism which targets to reduce the ICI at colliding RBs by employing power control strategy. The modelled system has adopted 7-cell hexagonal layout (2 coexistent-10MHz Evolved Universal Terrestrial Radio Access frequency division duplexing systems) with omnidirectional antennas at the centre of each cell, depicted in Fig.2. The RNN-CE is embedded inside the reference cognitive-eNodeB (C-eNodeB). $M C S$ and Powers $\left(P_{0}, P 1, P 2, P 3, P 4, P 5, P 6\right)$ are the configuration parameters or knobs of reference cell and adjacent eNodeB UEs, which are discussed in detail in Subsection-C and D. $\mathrm{C}$-eNodeB is responsible for monitoring and configuring the UE once it is attached and also the management of radio resources. In addition, make decisions on acceptable transmit power of UEs served by adjacent eNodeBs. UEs are responsible for the enforcement of decisions made by $\mathrm{C}$-eNodeB and sending environmental measurements back to C-eNodeB.

\section{B. Modelling Assumptions and Calculations}

The systems are $100 \%$ loaded with frequency reuse of $1 / 1$. The UEs are deployed randomly according to a uniform geographical distribution in the whole network region. Moreover, 


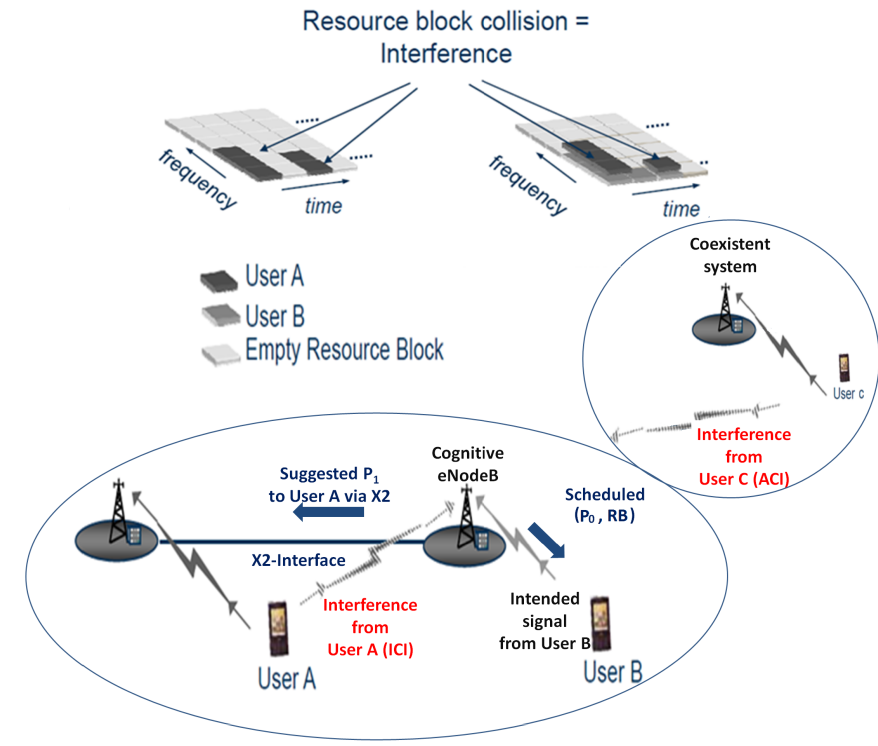

Fig. 1. ICI caused by collision between resource blocks which are used simultaneously by several users and corresponding scheduling based on the experienced CQI and interference in subsequent TTI

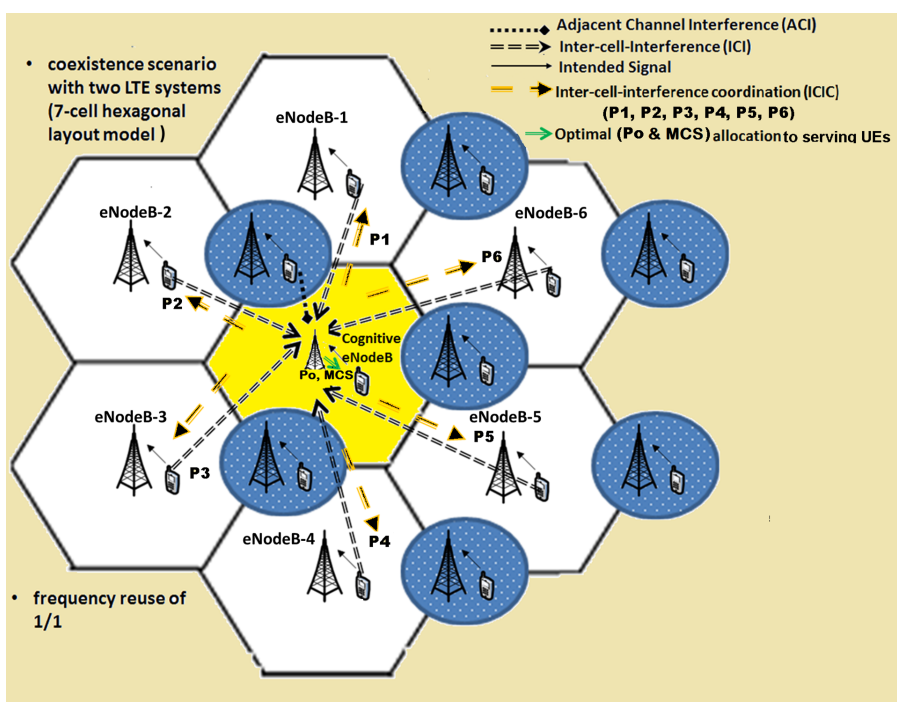

Fig. 2. Proposed System Model

wrap around technique is employed in order to remove the edge effects. The carrier frequencies of victim and external coexistent systems were set to $2000 \mathrm{MHz}$ and $2010 \mathrm{MHz}$ with inter-site distance of $750 \mathrm{~m}$. OFDMA urban macro propagation model is used. BS and UE antennas gains were assumed to be $15 \mathrm{dBi}$ and $0 \mathrm{dBi}$. $24 \mathrm{RBs}$ per $\mathrm{BS}$ and 3 per user were assumed i.e. 8 UEs per cell. In addition, log-normal shadowing variance: $10 \mathrm{~dB}$ with correlation; minimum coupling loss (MCL): $70 \mathrm{~dB}$; system bandwidth: $10 \mathrm{MHz}$; bandwidth of RB: $180 \mathrm{kHz}$; handover $(\mathrm{HO})$ margin: $3 \mathrm{~dB}$; thermal noise density: $-174 \mathrm{dBm} / \mathrm{Hz}$; BS noise figure: $5 \mathrm{~dB}$; UE min and max transmit power: $-30 \mathrm{dBm}$ to $24 \mathrm{dBm}$ were the system settings. The FPC settings, OFDMA LTE link-to-system level mapping, adjacent channel leakage ratio/unwanted spectrum mask were the same as given in Qualcomm STG(08)13 and 3GPP technical specification [17].

At the beginning of simulation (for every snapshot), UEs were randomly distributed throughout the system area and assigned a discrete speed value i.e. 0/3/30/100 kms./hr. The UEs get attached to the most appropriate BS depending on the HO margin, path-loss, antenna gain, and log-normal fading. The connected UEs (active) were scheduled for every snapshot and allocated a certain amount of resources according to the quality-of-service (QoS) requirement. Every BS goes through with all MSs on its served mobile list and try adding their requested sub-carriers until all MSs are served or number of available sub-carriers exceed the maximum limit. In the latter case, the BS discards the last MS and tries connecting the next mobile in line which may have less required number of sub-carriers. This is equivalent to modelling a round robin scheduler with a full buffer traffic model.

The signal-to-interference-noise ratio (SINR) and throughput for each UE with respect to link-to-system-level mapping is determined as follows:

$$
C(j, k)=P_{t}(j, k) * \text { pathloss }_{\text {effective }}\left(U E_{j, k}, B S_{j}\right)
$$

where $C j, k$ is the received power at $j^{\text {th }}$ serving C-eNodeB from the $k^{\text {th }} \mathrm{UE}, P_{t}$ is the transmit power of UE in $\mathrm{dBm}$, and pathloss effective $_{\text {is }}$ the effective path-loss which considered MCL as defined in [17].

The combined ICI and ACI at the victim reference cell is calculated as follows:

$$
I(j, k)=I_{\text {inter }}(j, k)+I_{\text {ext }}(j, k)+N_{t}(\text { thermal-noise })
$$

where $I_{\text {inter }}(j, k)$ is the ICI coming from the UEs of adjacent cells operating on same frequency sub-carriers and is calculated as follows:

$$
\begin{array}{r}
I_{\text {inter }}=\sum_{l \equiv 1, l \neq j}^{N_{\text {Cell }}} p_{t}(l, k) * \\
\text { pathloss }_{\text {effective }}\left(U E_{l, k}, B S_{j}\right)
\end{array}
$$

$I_{\text {ext }}(j, k)$ is the ACI coming from the UEs on adjacent channels in coexistent LTE system. ACI is the combination of $I_{\text {unwanted }}$ (unwanted emission in adjacent band) and $I_{\text {blocking }}$ (blocking effect of receiver) and is calculated as:

$$
I_{\text {ext }}=\sum_{m \equiv 1}^{N_{E x t C e l l}} \sum_{v \equiv 1}^{k} i R S S_{\text {blocking }}\left(U E_{m, v}, B S_{j}\right) *
$$

The bit-rate for all uplink users is collected as follows:

$$
\text { Bit }- \text { rate }=\frac{N_{s c_{p e r-U E}}}{N_{\text {total }-s c}}\left(x_{\frac{b p s}{H z}}\right)_{S I N R} \times B W_{M H z}
$$

where $N_{s c_{p e r-U E}}$ and $N_{t o t a l-s c}$ are the number of allocated sub-carriers to each UE and total number of sub-carriers available at each BS. The $x_{\frac{b p s}{H}}$ is the spectral efficiency with respect to calculated SNIR and $B W_{M H z}$ is the bandwidth. 


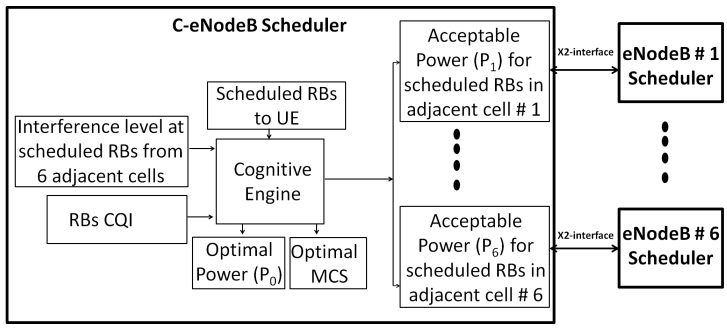

Fig. 3. UE scheduling example

\section{Scheduling framework}

Once the C-eNodeB scheduler selects the UE and assign RBs for uplink transmission, the embedded CE selects the optimal radio parameters (MCS and powers) based on CQI and interference on scheduled RBs in subsequent transmission time interval (TTI), such that the target SINR is achieved. In addition, suggest the optimal transmit powers of UEs served by adjacent eNodeBs, operating on same scheduled RBs via $\mathrm{X} 2$-interface (a communication interface between eNodeBs). This process is depicted in Fig. 3 for 7 cell hexagonal layout.

\section{CE Design}

The optimization framework is summarized in Fig.4, where the data is first collected for learning which helps GA based reasoning process to make optimised decisions. Fig.5 illustrates this further, where the learning entity with optimization is integrated. The learning module observes the channel $\mathrm{X}$ and estimates the performance $\mathrm{S}$ given radio configuration $\mathrm{Y}$. The vectors $\mathrm{X}, \mathrm{Y}$, and $\mathrm{S}$ are the training parameters coming from the radio. The radio communicates with optimizer the required objectives and current CQI. The optimizer then queries the learning module with considered $\mathrm{X}$ and $\mathrm{Y}$. The learning section returns the approximate performance of considered $\mathrm{X}$ and $\mathrm{Y}$ i.e. $\mathrm{P}(\mathrm{S} \mid \mathrm{X}, \mathrm{Y})$. Based on this report, the optimization section decides the optimal parameters.

The information, which is available to the cognitive controller, can be classified into three categories: environmental measurements (external factors affecting the reliability of communication), configuration parameters (tuning knobs which can be changed in an optimal way to achieve desired performance), and performance metric. After the careful observation that how different configuration parameters and environmental measurements are effecting the system performance, we feeded the following configuration parameters and environmental measurements into the RNN black-box. The justification of selecting these parameters and environmental measurements is evident through (1-5), where a clear relationship among SINR, bit-rate, transmit power of intended UE, and transmit power of interfering UE can be seen.

- Environmental measurements $(X)$ : we have considered SINR, ICI, and ACI as environmental measurements.

- Configuration parameters $(X)$ : considered parameters are available channels $(\mathrm{RBs})$, transmit power $\left(P_{0}\right)$, and MCS of all UEs served by C-eNodeB and the transmit

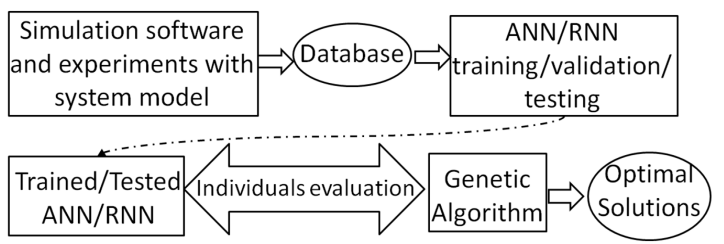

Fig. 4. Optimization framework

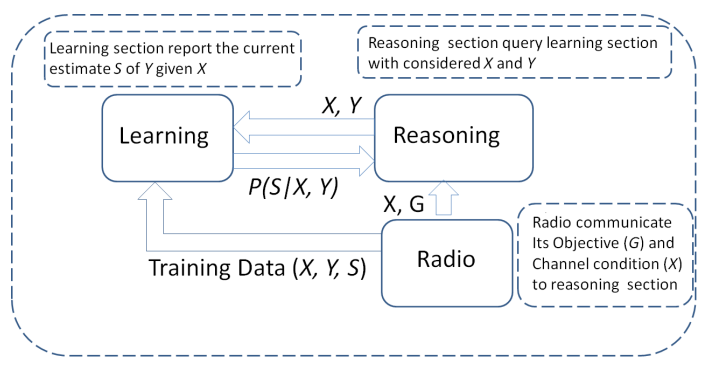

Fig. 5. Simplified cognitive engine operation

powers $\left(P_{1}, P_{2}, P_{3}, P_{4}, P_{5}, P_{6}\right)$ of all UEs served by adjacent 6-eNodeBs.

- Performance metric (Y): expected throughput for each $\mathrm{C}$-eNodeB UE as a performance measure is considered.

The UEs configuration parameters and environmental measurements as defined above are the inputs of RNN black-box and the performance metric is the output. The UEs configuration parameters and environmental measurements as defined above are the inputs of RNN black-box and the performance metric is the output. It is to be noted that the required input parameters are by default available at the cognitive eNodeB where the CE is embedded, with no extra effort required. Moreover, the scheduling process require only one way communication/coordination between eNodeBs, which is from cognitive eNodeB to adjacent eNodeBs. With a feature set $X$, label set $Y$, and $\mathrm{n}$ training samples $T=\left(\left(x_{1}, y_{1}\right), . .,\left(x_{n}\right.\right.$, $\left.\left.y_{n}\right)\right) \epsilon\left(\begin{array}{lll}X & x & Y\end{array}\right)^{n}$, a ML algorithm creates a mapping $A: X \rightarrow Y$ from features to labels and predicts the labels for new samples.

\section{Random Neural Network}

RNN, a machine learning technique, made up of highly interconnected processing elements called as neurons, processes the information by their state response and learn from examples. The main objective of the RNN model is to transform the inputs into meaningful outputs, learn the input-output relationship, and offer viable solutions to unseen problems (a generalization capability). RNNs were first developed by Gelenbe [18] as a new modified class of ANNs, representing the transmission of signals in a very similar form to biological neural networks, but offers more benefits and cope the limitations of ANNs. In RNN, the neuron exchanges the signal in the form of spikes. The potential $(k)$ of each neuron represents its state that increases/decreases with respect to an incoming signal. A neuron $u$ can receive positive/negative exogenous signals, modelled as Poisson arrival streams of rates $\Lambda_{u}$ and 
$\lambda_{u}$ respectively. If a neuron receives an excitatory signal (+1), its potential increases and correspondingly decreases upon receiving inhibitory signal $(-1)$. When the potential of neuron is equal to zero $\left(k_{i}=0\right)$, it is in idle state and when $\left(k_{i}>0\right)$, the neuron is excited. In the state of excitation, the neuron fires an excitatory spike that goes from neuron $u$ to $v$. In that case, the potential of neuron $u$ decreases by one, whereas potential of neuon $v$ increases by one. When neuron fires inhibitory spike, the potential of both neuron decreases by one. The firing is according to the Poisson process represented by the synaptic weights $w_{i j}^{+}=r P_{i j}^{+}$and $w_{i j}^{-}=r P_{i j}^{-}$, where $P_{i j}^{+}$and $P_{i j}^{-}$are the probabilities of excitatory and inhibitory signals and $r$ is the spikes firing rate. The $w_{i j}^{+}$and $w_{i j}^{-}$can be seen as the positive and negative rates of signal transmissions and these are the typical interconnections weights of a neural network that RNN learns through the process of learning or training.

The average rate of +ive signals at neuron $i\left(\lambda_{i}^{+}\right)$, average rate of -ive signals at neuron $i\left(\boldsymbol{\lambda}_{\boldsymbol{i}}^{-}\right)$, and the probability that neuron $i$ is excited $\left(\boldsymbol{q}_{i}\right)$, are calculated using the following equations:

$$
\begin{gathered}
\lambda_{i}^{+}=\Lambda_{i}+\sum_{j=1}^{n} q_{j} w_{i j}^{+} \\
\lambda_{i}^{-}=\lambda_{i}+\sum_{j=1}^{n} q_{j} w_{i j}^{-} \\
q_{i}=\frac{\lambda_{i}^{+}}{r_{i}+\lambda_{i}^{-}}
\end{gathered}
$$

1) Network Behaviour in Steady State: if $0 \leq q_{i} \leq 1$ for $i=1,2,3 \ldots, n$ then the stationary joint probability of network $p(k, t)=p_{r}=[k(t)=k]$ can be written as:

$$
p(k)=\Pi_{i=1}^{n}\left(1-q_{i}\right) q_{i}^{k}
$$

2) Network Stability: The network is stable, if the signal potential increases with bounds. Stability can be guaranteed if a unique solution to non-linear equations (6-8) exists. The existence of solution implies its uniqueness because for any neuron $i$, it is not possible to have two different $\boldsymbol{q}_{\boldsymbol{s}}$. Moreover, in feed-forward RNN, the solution always exists since $q$ for every neuron is computed from the values of neurons on preceding layer [11].

3) RNN Training: The goal of training is to learn desired system behaviour and adjust the network parameters (interconnections weights) to map (learn) the input-output relationship and minimize the mean square error (MSE). The standard GD training algorithm was proposed in [19] which has been used in this paper. In [14][15], we also implemented AIW-PSO, DE and GA based learning algorithms. However, in general, there is a trade-off among learning accuracy, convergence time, calculation time, and computational complexity. Further indepth details and procedure for GD, AIW-PSO, DE, and GA learning algorithms is presented in [14][15].
TABLE I

ANN vs. RNN TRAINING PERformanCE

\begin{tabular}{|l|l|l|l|l|}
\hline Methods & $\begin{array}{l}\text { Achieved } \\
\text { MSE }\end{array}$ & $\begin{array}{l}\text { Average } \\
\text { time/iter } \\
(\mathrm{s})\end{array}$ & $\begin{array}{l}\text { Total } \\
\text { training } \\
\text { time (s) }\end{array}$ & $\begin{array}{l}\text { Number } \\
\text { of itera- } \\
\text { tions }\end{array}$ \\
\hline $\begin{array}{l}\text { ANN- } \\
\text { GD }\end{array}$ & 2.88 E-03 & 0.00581 & 33 & 10000 \\
\hline $\begin{array}{l}\text { ANN- } \\
\text { LM }\end{array}$ & 1.37 E-06 & 0.0411 & 57 & 313 \\
\hline $\begin{array}{l}\text { RNN- } \\
\text { GD }\end{array}$ & 6.9 E-04 & 4.62 & 566 & 121 \\
\hline
\end{tabular}

\section{Performance Evaluation}

\section{A. Simulation Assumptions}

The simulations have been performed using SEAMCATLTE simulator [20], Eclipse, and Matlab. SEAMCAT was used to accurately model the CR environment of our complex cognitive radio network and to build the dataset for training. Eclipse was used as a Java editor in order to extract the required parameters from the modelled scenario for post processing. The trace file was obtained by running the system model using SEAMCAT online available source code. However, we further developed the obtained source code for post processing and added some new classes and functions. The trace file consists the required configuration parameters, environmental measurements, and performance metric statistics for specified number of instances. Matlab was used for training and validation of neural networks. Learning rate for GD and LM was set to 0.01 and 0.001 . More RNN simulation settings are the same as given in [21]. The network was trained/validated with the dataset of 4000 samples. A subset of dataset was used to train the neural network (NN) and rest of the data was used to compare the prediction performance of trained $\mathrm{NN}$ in face of new environment condition (only for Test-Case I), which is elaborated further in $\mathrm{CE}$ testing subsection.

\section{B. CEs Training}

As described in Section II that the proposed CE consists two processes. One, the learning process and second, the process of reasoning. The learning process is the core of decision making process; therefore, its role is critical and the quality of decision making is completely dependent on the learning quality. The performance of learning process was evaluated using MSE, which represents how well the CE has learnt the system behaviour. Once, the system behaviour is learnt, the $\mathrm{CE}$ characterizes the achievable performance of possible actions i.e., the configuration parameters with respect to current situation, and then selects the most appropriate configuration parameters. The main aim of CE training is to achieve the least possible MSE in less training time.

In training, different number of neurons, hidden layers and epochs were tried. The best performed RNN/ANN structures were 1 hidden layer with 11 neurons and 1 hidden layer with 20 neurons. The training performance is illustrated in Table-I where the least MSE achieved by ANN-GD is $2.88 \mathrm{E}-03$ in $10 \mathrm{~K}$ iterations (33 sec), ANN-LM achieved $1.37 \mathrm{E}-06$ in 313 
TABLE II

ANN vs. RNN Testing Performance

\begin{tabular}{|l|l|l|l|l||l|}
\hline Test-Cases & Scenario & Configurations & MSE \\
\cline { 2 - 6 } & $\begin{array}{l}\text { ANN-GD } \\
\text { ANN-LM }\end{array}$ & RNN-GD \\
\hline I & $\begin{array}{l}\text { Macro-OFDMA Urban propagation } \\
\text { channel }\end{array}$ & Same as used for training & $2.67 \mathrm{E}-03$ & $1.31 \mathrm{E}-06$ \\
\hline $\begin{array}{l}\text { Macro-OFDMA Urban propagation } \\
\text { channel }\end{array}$ & Change in active no. of UEs & $1.79 \mathrm{E}-02$ & $1.52 \mathrm{E}-02$ \\
\hline III & $\begin{array}{l}\text { Macro-OFDMA Suburban propaga- } \\
\text { tion channel }\end{array}$ & $\begin{array}{l}\text { Change in propagation channel from Urban to } \\
\text { Suburban (Macro OFDMA) }\end{array}$ & $1.5 \mathrm{E}-02$ & $1.7 \mathrm{E}-02$ \\
\hline IV & $\begin{array}{l}\text { Macro-OFDMA Rural propagation } \\
\text { channel }\end{array}$ & $\begin{array}{l}\text { Change in propagation channel from Urban to } \\
\text { Rural (Macro OFDMA) }\end{array}$ & $1.8 \mathrm{E}-02$ & $2.15 \mathrm{E}-03$ \\
\hline V & $\begin{array}{l}\text { Extended Hata Urban propagation } \\
\text { channel }\end{array}$ & $\begin{array}{l}\text { Change in propagation channel from Urban to } \\
\text { Extended Hata }\end{array}$ & $1.4 \mathrm{E}-01$ & $1.08 \mathrm{E}-01$ & $2.1 \mathrm{E}-02$ \\
\hline VI & $\begin{array}{l}\text { Macro-OFDMA Urban propagation } \\
\text { channel }\end{array}$ & $\begin{array}{l}\text { Same as system model but with fixed training } \\
\text { time of } 2 \text { sec }\end{array}$ & $1.34 \mathrm{E}-01$ & $1.28 \mathrm{E}-01$ & $1.60 \mathrm{E}-02$ \\
\hline
\end{tabular}

iterations (57 sec), and RNN-GD achieved the MSE of 6.9 E-04 in 121 iterations $(566 \mathrm{sec})$. The training performance of ANN in terms of accuracy and training time was better than RNN-GD but ANN could only perform well for the trained cases, which is discussed in the following Subsection.

\section{CEs Testing under CE Design Requirements}

1) Long-Term Learning: The CEs were assumed to be working on a critical mission, where they do not have a privilege of retraining upon extreme propagation environment change. For such cases, long-term learning capability is essential which enables radio to adapt optimal radio parameters in a completely unknown scenario. To investigate the long-term learning, the CEs were tested in different wireless environments for which they were not trained. Table-II illustrates six testing conditions along with the decision making accuracy of CEs. The testing conditions varied in urban, suburban, and rural propagation channels with different assumptions such as indoor, outdoor transmitter-receiver location, wall losses, losses between adjacent floors of the building, empirical parameters etc. Moreover, voice traffic variations with the assumption of training time constraints were also considered. Variation in electromagnetic environment affected the whole system in terms of average system throughput loss, average system SINR, internal/external interferences etc., which examined the decision making ability of the engines. In TestCase I, ANN-LM outperformed RNN-GD mainly because of the testing in trained scenario. In all other test cases, as the electromagnetic environment changed, subsequently the performance of ANN started decreasing. In contrast, RNNGD with its long-term learning remained 53.88\% - 87.53\% accurate in making optimal and reliable decisions. A summary of all test cases is depicted in Fig.6, where the out-performance of RNN based CE is evident.

2) Decision Making Speed at Run-Time: In real-time $\mathrm{CE}$ applications, fast decision making means that $\mathrm{CE}$ can respond quickly upon severe wireless environment change. As a core optimization algorithm, we not only require the decision accuracy but also the response speed. In training phase, the performance of ANN was found to be faster but during run-time the RNN-GD outperformed ANN-LM/GD

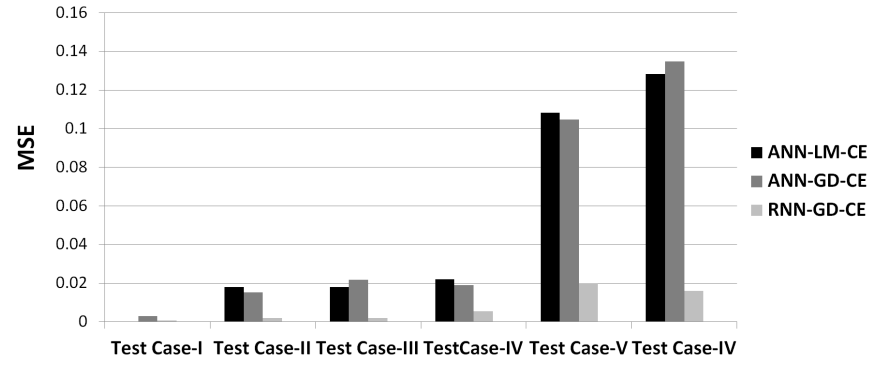

Fig. 6. CEs testing in six different Test-Cases for the investigation of long-term learning capability

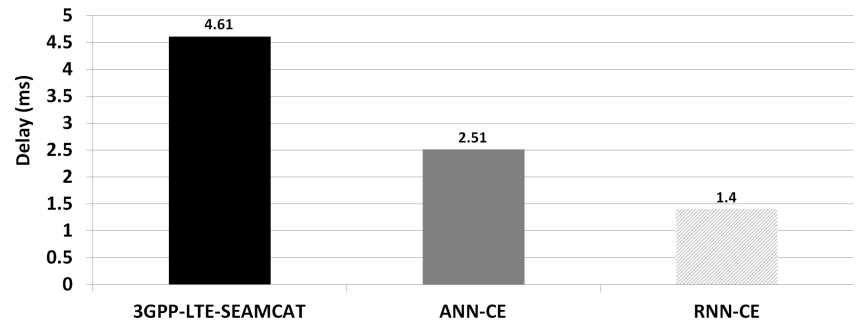

Fig. 7. LTE uplink end-to-end scheduling delay comparison

in total calculation time (decision making speed). This is mainly because of RNNs 3-level architecture in which the computation of output during run-time is extremely fast. As its non-linear system equations computes the output values of input neurons directly from its input and similarly for hidden neurons output. For more details with mathematical proofs, see [18][19][11]. The decision making speed was tested by embedding ANN/RNN based controller within eNodeB, replacing the traditional LTE optimization algorithms for UEs parameters settings. The decision making delays are illustrated in Fig.7, where RNN based CE outperformed SEAMCAT and ANN.

\section{Performance Gain}

In Fig. 8, 9, and 10, we show the performance gain of proposed approach as compared to ANN and FPC methods. Fig. 8 and Fig. 9 are showing the CDF of UEs throughput 


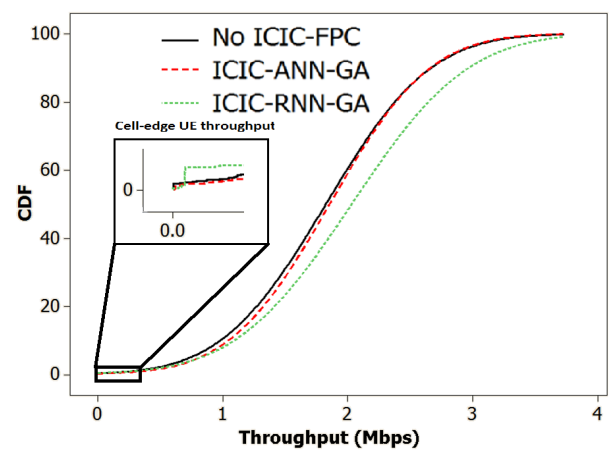

Fig. 8. User throughput (reference cell)

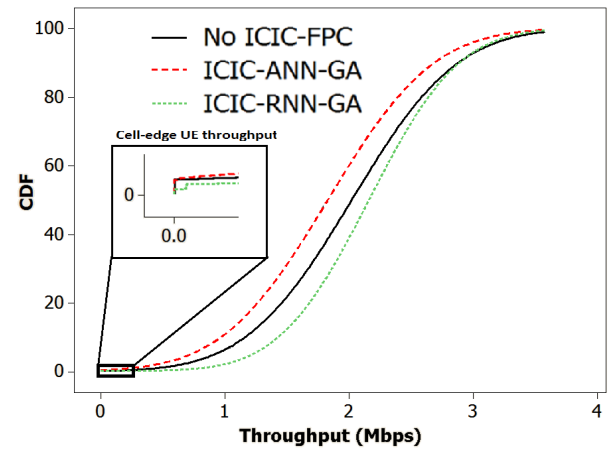

Fig. 9. User throughput (system)

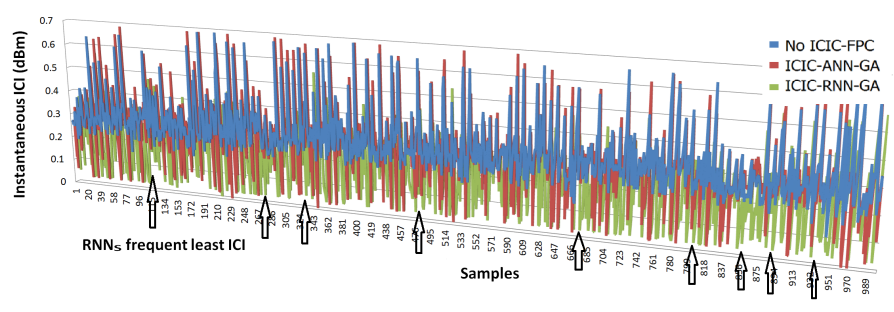

Fig. 10. Instantaneous ICI (normalized)

in reference cell and over the entire system respectively. Similarly, Fig. 10 depicts the instantaneous received ICI at reference cell. Clearly, the RNN based optimization framework has outperformed both FPC and ANN. Therefore, it is evident that for proposed optimization framework, RNN is the optimal choice.

\section{Conclusion}

In this article, we presented an RNN based cognitive eNodeB for RRM and ICIC. The focus of this work was mainly to enhance the learning capability of designed $\mathrm{CE}$ and analyse its performance taking into account the $\mathrm{CE}$ design requirements. Going beyond the discussion, we envision advanced AI/ML techniques such as RNN being implemented in hardware and real CR systems. The proposed RNN based CE achieved the essential CE design features, which other AI/ML approaches could not. We believe that inherent RNN properties ideally place it as a powerful tool for CR. There are further AI/ML techniques we have not considered and more advanced versions of the RNN, both in structure of the neural network and also training algorithms. We believe the use of these methods applied to complex CR problems are one of the best tools.

\section{REFERENCES}

[1] M. Boussif, N. Quintero, F. D. Calabrese, C. Rosa, and J. Wigard, "Interference based power control performance in lte uplink," in Wireless Communication Systems. 2008. ISWCS'08. IEEE International Symposium on. IEEE, 2008, pp. 698-702.

[2] M. Bkassiny, Y. Li, and S. K. Jayaweera, "A survey on machine-learning techniques in cognitive radios," Communications Surveys \& Tutorials, IEEE, vol. 15, no. 3, pp. 1136-1159, 2013.

[3] A. S. Hamza, S. S. Khalifa, H. S. Hamza, and K. Elsayed, "A survey on inter-cell interference coordination techniques in ofdma-based cellular networks," Communications Surveys \& Tutorials, IEEE, vol. 15, no. 4, pp. 1642-1670, 2013.

[4] A. Attar, V. Krishnamurthy, and O. N. Gharehshiran, "Interference management using cognitive base-stations for umts lte," Communications Magazine, IEEE, vol. 49, no. 8, pp. 152-159, 2011.

[5] H. I. Volos and R. M. Buehrer, "Cognitive radio engine training," Wireless Communications, IEEE Transactions on, vol. 11, no. 11, pp. 3878-3889, 2012.

[6] A. Galindo-Serrano and L. Giupponi, "Distributed q-learning for aggregated interference control in cognitive radio networks," Vehicular Technology, IEEE Transactions on, vol. 59, no. 4, pp. 1823-1834, 2010.

[7] M. Brehm and R. Prakash, "Proactive resource allocation optimization in lte with inter-cell interference coordination," Wireless networks, vol. 20, no. 5, pp. 945-960, 2014

[8] S. Deb and P. Monogioudis, "Learning based uplink interference management in $4 \mathrm{~g}$ lte cellular systems," IEEE Transaction, arXiv preprint arXiv:1309.2543, 2013.

[9] M. Simsek, M. Bennis, and I. Guvenc, "Learning based frequencyand time-domain inter-cell interference coordination in hetnets," IEEE Transaction, arXiv preprint arXiv:1411.5548, 2014

[10] V. Poulkov, P. Koleva, O. Asenov, and G. Iliev, "Combined power and inter-cell interference control for lte based on role game approach," Telecommunication Systems, vol. 55, no. 4, pp. 481-489, 2014.

[11] S. Timotheou, "The random neural network: a survey," The computer journal, vol. 53, no. 3, pp. 251-267, 2010 .

[12] A. Adeel, H. Larijani, and A. Ahmadinia, "Performance analysis of random neural networks in lte-ul of a cognitive radio system," in $1 s t$ IEEE International Workshop on Cognitive Cellular Systems, IEEE CCS 2014, Rhine River, Germany, Sept 2-4, 2014.

[13] Adeel, H. Larijani, and A. Ahmadinia, "Efficient use of random neural networks for cognitive radio system in lte-ul," in in Proc. IEEE International Symposium on Wireless Communication Systems, ISWCS 2014 Barcelona, Spain, August 26-29, 2014.

[14] A. Adeel, H. Larijani, A. Javed, and A. Ahmadinia, "Critical analysis of learning algorithms in random neural network based cognitive engine for lte systems," in IEEE Vehicular Technology Conference (VTC), 2015 (accepted for publication).

[15] Adeel, H. Larijani, A. Javed, and A. Ahmadinia, "Random neural network based power controller for inter-cell interference coordination in lte-ul," in IEEE ICC 2015 Workshop on Advances in Software Defined and Context Aware Cognitive Networks, 2015, (accepted for publication).

[16] G. Fodor, C. Koutsimanis, A. Rácz, N. Reider, A. Simonsson, and W. Müller, "Intercell interference coordination in ofdma networks and in the 3gpp long term evolution system," Journal of Communications, vol. 4, no. 7, pp. 445-453, 2009.

[17] 3GPP. (2009) Technical specification group radio access network, eutra, rf system scenarios (release 9).

[18] E. Gelenbe, "Random neural networks with negative and positive signals and product form solution," Neural computation, vol. 1, no. 4, pp. 502$510,1989$.

[19] Gelenbe, "Learning in the recurrent random neural network," Neural Computation, vol. 5, no. 1, pp. 154-164, 1993.

[20] J. Deloziere, A. Toury, and M. Le Devendec, "Seamcat user manual," ERO, Feb, 2010.

[21] H. Abdelbaki, "Random neural network simulator (rnnsim) v. 2," 1999. 